\title{
Erratum to: Tropical Animal Health and Production, Vol. 45 No. 1, January 2013
}

Verena Penning

Published online: 22 February 2014

(C) Springer Science+Business Media Dordrecht 2014

Erratum to: Trop Anim Health Prod

DOI 10.1007/s11250-012-0193-2

DOI 10.1007/s11250-012-0224-z

DOI 10.1007/s11250-012-0223-0

DOI 10.1007/s11250-012-0222-1

DOI 10.1007/s11250-012-0221-2

DOI 10.1007/s11250-012-0220-3

DOI 10.1007/s11250-012-0219-9

DOI 10.1007/s11250-012-0218-x

DOI 10.1007/s11250-012-0217-y

DOI 10.1007/s11250-012-0216-z

DOI 10.1007/s11250-012-0213-2

DOI 10.1007/s11250-012-0212-3

DOI 10.1007/s11250-012-0211-4

DOI 10.1007/s11250-012-0210-5

DOI 10.1007/s11250-012-0198-x

DOI 10.1007/s11250-012-0197-y

DOI 10.1007/s11250-012-0196-z

DOI 10.1007/s11250-012-0195-0

DOI 10.1007/s11250-012-0194-1

DOI 10.1007/s11250-012-0192-3

DOI 10.1007/s11250-012-0191-4

DOI 10.1007/s11250-012-0190-5

DOI 10.1007/s11250-012-0189-y

DOI 10.1007/s11250-012-0188-z

DOI 10.1007/s11250-012-0187-0

DOI 10.1007/s11250-012-0186-1

DOI 10.1007/s11250-012-0185-2

The online version of the original article can be found under http://dx.doi. org/10.1007/s11250-012-0193-2.

V. Penning $(\bowtie)$

Life Sciences, Biomedicine Europe II, Springer, Tiergartenstr. 17, 69121 Heidelberg, Germany

e-mail: Verena.Penning@springer.com
DOI 10.1007/s11250-012-0184-3

DOI 10.1007/s11250-012-0183-4

DOI 10.1007/s11250-012-0182-5

DOI 10.1007/s11250-012-0181-6

DOI 10.1007/s11250-012-0180-7

DOI 10.1007/s11250-012-0179-0

DOI 10.1007/s11250-012-0178-1

DOI 10.1007/s11250-012-0177-2

DOI 10.1007/s11250-012-0176-3

DOI 10.1007/s11250-012-0175-4

DOI 10.1007/s11250-012-0174-5

DOI 10.1007/s11250-012-0173-6

DOI 10.1007/s11250-012-0172-7

DOI 10.1007/s11250-012-0254-6

DOI 10.1007/s11250-012-0246-6

DOI 10.1007/s11250-012-0235-9

DOI 10.1007/s11250-012-0215-0

Tropical Animal Health and Production, Vol. 45 No. 1, January 2013: Owing to an unfortunate technical problem, the publication month is falsely displayed as December 2012 in the online edition of this issue; the print edition shows the correct publication month: January 2013. All articles in the issue Tropical Animal Health and Production, volume 45 issue 1, 2013 are affected. We apologize for the incorrect presentation.

The DOIs of the articles in the issue are listed below.

$10.1007 / \mathrm{s} 11250-012-0215-0$

$10.1007 / \mathrm{s} 11250-012-0235-9$

$10.1007 / \mathrm{s} 11250-012-0246-6$

$10.1007 / \mathrm{s} 11250-012-0254-6$

$10.1007 / \mathrm{s} 11250-012-0172-7$

$10.1007 / \mathrm{s} 11250-012-0173-6$

$10.1007 / \mathrm{s} 11250-012-0174-5$

$10.1007 / \mathrm{s} 11250-012-0175-4$ 
10.1007/s11250-012-0176-3

$10.1007 / \mathrm{s} 11250-012-0177-2$

$10.1007 / \mathrm{s} 11250-012-0178-1$

$10.1007 / \mathrm{s} 11250-012-0179-0$

$10.1007 / \mathrm{s} 11250-012-0180-7$

$10.1007 / \mathrm{s} 11250-012-0181-6$

$10.1007 / \mathrm{s} 11250-012-0182-5$

$10.1007 / \mathrm{s} 11250-012-0183-4$

$10.1007 / \mathrm{s} 11250-012-0184-3$

$10.1007 / \mathrm{s} 11250-012-0185-2$

$10.1007 / \mathrm{s} 11250-012-0186-1$

$10.1007 / \mathrm{s} 11250-012-0187-0$

$10.1007 / \mathrm{s} 11250-012-0188-\mathrm{z}$

$10.1007 /$ s11250-012-0189-y

$10.1007 / \mathrm{s} 11250-012-0190-5$

$10.1007 / \mathrm{s} 11250-012-0191-4$

$10.1007 / \mathrm{s} 11250-012-0192-3$

$10.1007 / \mathrm{s} 11250-012-0193-2$ 10.1007/s11250-012-0194-1

10.1007/s11250-012-0195-0

10.1007/s11250-012-0196-Z

10.1007/s11250-012-0197-y

10.1007/s11250-012-0198-x

10.1007/s11250-012-0210-5

$10.1007 / \mathrm{s} 11250-012-0211-4$

$10.1007 / \mathrm{s} 11250-012-0212-3$

$10.1007 / \mathrm{s} 11250-012-0213-2$

$10.1007 / \mathrm{s} 11250-012-0216-\mathrm{z}$

$10.1007 / \mathrm{s} 11250-012-0217-\mathrm{y}$

$10.1007 / \mathrm{s} 11250-012-0218-\mathrm{x}$

$10.1007 / \mathrm{s} 11250-012-0219-9$

$10.1007 / \mathrm{s} 11250-012-0220-3$

10.1007/s11250-012-0221-2

$10.1007 / \mathrm{s} 11250-012-0222-1$

$10.1007 / \mathrm{s} 11250-012-0223-0$

$10.1007 / \mathrm{s} 11250-012-0224-\mathrm{z}$ 